\title{
Multiple sequence elements in the c-fos promoter mediate induction by cAMP
}

\author{
Tobe M. Fisch, ${ }^{1}$ Ron Prywes, ${ }^{2}$ M. Celeste Simon, ${ }^{3}$ and Robert G. Roeder ${ }^{1}$ \\ ${ }^{1}$ Laboratory of Biochemistry and Molecular Biology and ${ }^{2}$ Laboratory of Molecular Cell Biology, The Rockefeller University, \\ New York, New York 10021 USA
}

\begin{abstract}
Induction of c-fos by cyclic AMP in NIH-3T3 cells is distinct from induction by serum. Whereas induction by serum is mediated by the serum response element (SRE), induction by cAMP does not require this element. In fact, no single sequence element in the c-fos promoter/enhancer is stringently required for the cAMP response. Rather, multiple sequence elements in the c-fos promoter/enhancer can mediate induction by cAMP independently. These elements are: (1) the region from -72 to -54 , which contains a binding site for a cellular activating transcription factor (ATF); (2) the region from -225 to -99 ; (3) the region from -303 to -281 , which is homologous to the consensus binding site for the transcription factor AP1; and (4) the region from -317 to -298 , which contains the SRE. These sequence elements convey cAMP inducibility when fused to the cAMP-inresponsive 'minimal' fos promoter $(-53$ to +42$)$. In addition, the c-fos regions from -700 to -63 and from -71 to -48 can confer CAMP inducibility to a heterologous promoter.
\end{abstract}

[Key Words: c-fos; serum response element; cAMP; activating transcription factor]

Received August 9, 1988; revised version accepted December 12, 1988.

Transcription of the c-fos gene is induced rapidly and transiently by a wide variety of agents that influence the growth or differentiation state of the cell (reviewed in Curran 1988). The induction of c-fos may be an early, critical step in a cascade of gene expression triggered by cell surface signals, since c-fos gene products themselves appear to influence the expression of other cellular genes (Setoyama et al. 1986; Distel et al. 1987; Franza et al. 1988; Rauscher et al. 1988a,b). As a consequence, much effort has been directed toward an elucidation of the mechanisms of transcriptional activation of c-fos. One approach has been to identify sequence elements that are crucial for mediating induction by various agents, and, especially, to determine whether the different inducers of c-fos transcription use discrete or convergent pathways. Perhaps surprisingly, many of the inducers studied have been found to converge on a single pathway involving the so-called serum response element (SRE), a twenty base-pair dyad symmetry element located between -317 to -298 (relative to the c-fos mRNA cap site). SRE can mediate induction by serum, epidermal growth factor (EGF), phorbol esters such as 12 - $O$-tetradecanoyl phorbol-13-acetate (TPA), c-sis protein, and insulin (Treisman 1985; Gilman et al. 1986; Fisch et al. 1987; Greenberg et al. 1987; Stumpo et al. 1988). The calcium ionophore A23187 is the only one of the inducers of c-fos transcription which has been shown thus

Current addresses: ${ }^{2}$ Department of Biological Sciences, Columbia University, New York, New York 10027 USA; ${ }^{3}$ Howard Hughes Medical Institute, Children's Hospital, Division of Hematology and Oncology, Boston, Massachusetts 02115 USA. far to require sequence elements outside the enhancer for c-fos induction (Fisch et al. 1987).

Cyclic AMP is another agent that induces c-fos rapidly and transiently in a variety of cell types (Greenberg et al. 1985; Kruijer et al. 1985; Bravo et al. 1987; Gilman 1988). Experimental observations have suggested that this agent might induce c-fos through a pathway distinct from that used by phorbol esters and serum. For instance, long-term incubation of cells with phorbol esters, which downregulates protein kinase $\mathrm{C}$ (Nishizuka 1986), abolished induction of c-fos expression by TPA and reduced induction by serum and c-sis protein (Bravo et al. 1987; Gilman 1988). In contrast, such treatment had no effect on the induction of c-fos by cAMP, which activates the distinct cAMP-dependent protein kinase A (Krebs and Beavo 1979; Bravo et al. 1987; Gilman 1988).

The fact that common sequence elements distinct from the SRE are found in the c-fos promoter and in the promoters of other genes whose transcription is induced by cAMP hinted that sequence elements besides the SRE might be important in regulating cAMP-induced c-fos expression (Comb et al. 1986; Montminy et al. 1986). Studies of these genes have identified several sequence elements that are thought to mediate cAMP induction. One element, the CRE (cAMP response element), is found in the rat somatostatin and human vasoactive intestinal polypeptide genes and includes the eight base pair palindrome 5'-TGACGTCA-3' (Montminy et al. 1986, 1987; Tsukada et al. 1987). A sequence in the promoter of the human preproenkephalin gene has been implicated in the transcriptional induction of that gene 
by cAMP, and bears homology to the consensus binding sites for the transcription factors AP1 and AP4 (Comb et al. 1986; Mermod et al. 1988). The transcription factor AP2, along with its consensus binding sequence, has also been implicated in cAMP-inducible transcription, notably of the human metallothionein IIA gene (Imagawa et al. 1987). Homologies to all of these sequence elements can be found in the human c-fos promoter, and it seemed likely that at least some of these elements might be involved in c-fos induction by cAMP. To investigate this question we created mutants in the promoter of the human c-fos gene and analyzed their inducibility by cAMP following transfection into NIH-3T3 cells.

\section{Results}

cAMP induces $c$-fos rapidly and transiently in NIH-3T3 cells

As shown in Figure 1, $1 \mathrm{~mm}$ 8-Br cAMP-induced expression of c-fos in serum-starved NIH-3T3 cells. Induction of c-fos mRNA varied from 10-fold to greater than 20fold in different experiments and was maximal by 30 min after addition of 8-Br cAMP; c-fos mRNA declined to prestimulation levels by $3 \mathrm{hr}$ after addition of cAMP. These kinetics were very different from those observed in mouse macrophage cell lines, where c-fos expression remained elevated up to $6 \mathrm{hr}$ after addition of cAMP (Bravo et al. 1987). Generally, we observed that induction of c-fos in starved NIH-3T3 cells by cAMP was sev-

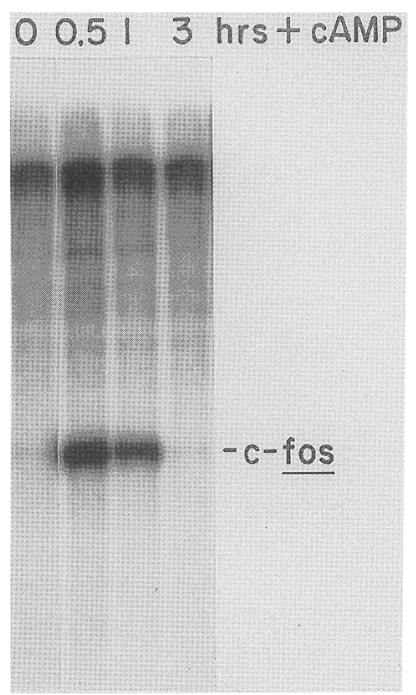

Figure 1. Kinetics of induction of c-fos mRNA in NIH-3T3 cells by $8-\mathrm{Br}$ cAMP. NIH-3T3 cells were grown to confluence in medium containing $10 \%$ bovine calf serum and then incubated for $24 \mathrm{hr}$ in medium containing $0.5 \%$ bovine calf serum. Parallel dishes of cells were then left untreated or treated for $0.5,1$, or $3 \mathrm{hr}$ with $1 \mathrm{mM} 8$-Br cAMP. Total cellular RNA (20 $\mu \mathrm{g}$ ) was analyzed for $\mathrm{c}$-fos transcripts by RNase protection mapping as described in Materials and methods. The position of the c-fosspecific protected fragment is indicated. eralfold lower than induction by $10 \%$ bovine calf serum (data not shown). While 8-Br cAMP-induced c-fos expression 10 - to 20 -fold, dibutyryl cAMP $(0.5$ to $10 \mathrm{mM})$ had no effect. However, treatment with other agents that raise cellular cAMP levels, such as $0.5 \mathrm{~mm}$ isobutyl methyl xanthine (IMX) and $10 \mathrm{~mm}$ forskolin, induced c-fos expression comparably to 8-Br cAMP (data not shown; methods based on Comb et al. 1986; Gilman 1988). Although we have not performed nuclear run-on analyses in cAMP-induced NIH-3T3 cells, the results presented below indicate strongly that the CAMP induction is transcriptional, as it has been shown for cAMP induction of c-fos in rat pheochromocytoma cells (Greenberg et al. 1985).

\section{The SRE is not required for induction of $c$-fos by $C A M P$}

SRE has been shown previously to mediate transcriptional induction of c-fos by a variety of agents. We tested whether SRE is also required for induction of c-fos by cAMP following transfection of fosCAT fusion genes into NIH-3T3 mouse fibroblasts. As described previously, the fosCAT constructs contain parts of the human c-fos gene, extending from variable 5 ' sites to position +42 , fused to the coding region of the bacterial gene for chloramphenicol acetyltransferase (CAT) (Fisch et al. 1987). Most of the constructs used in this study are diagramed in Figure 2. The plasmid pRSVCAT (Gorman et al. 1982) was included in every transfection as an internal control for transfection efficiency; in our assays, the expression of pRSVCAT was not affected by treatment with cAMP. Thus, induction of a given 'test' gene by cAMP was always normalized to expression of the pRSVCAT plasmid in transfected cells. In our initial experiments (Figs. 3-5, 6A), we 'starved' the transfected cells starting at about $16 \mathrm{hr}$ after transfection by incubating them for 24 to $28 \mathrm{hr}$ in medium containing $0.5 \%$ calf serum. At this point, the cells were treated for 30 min with $1 \mathrm{~mm} 8-\mathrm{Br}$ cAMP (or with $10 \%$ bovine calf serum) and total cellular RNA was isolated as described previously (Fisch et al. 1987). We found subsequently that, while serum starvation greatly increased the magnitude of induction by serum, it had no effect on the magnitude of induction of endogenous mouse c-fos or transfected fosCAT fusion genes by cAMP (data not shown). Therefore, in our later experiments, we isolated RNA $24-28 \mathrm{hr}$ after transfection, omitting starvation in low-serum media.

To analyze RNA harvested from the transfected cells, we used the S1 nuclease protection assay described previously (Fisch et al. 1987). Briefly, transcripts derived from the fosCAT fusion genes could be distinguished on the basis of size from pRSVCAT transcripts by use of an $S 1$ probe made from the coding and $5^{\prime}$ flanking region of the fosCAT hybrid gene. A 300 nucleotide fragment extending to the $5^{\prime}$ end of the fosCAT transcript was protected from S1 digestion, while only a 250 nucleotide fragment corresponding to the CAT portion of the pRSVCAT transcript was protected. Figure 3 shows the results of an S1 analysis of an experiment in which 
Fisch et al.

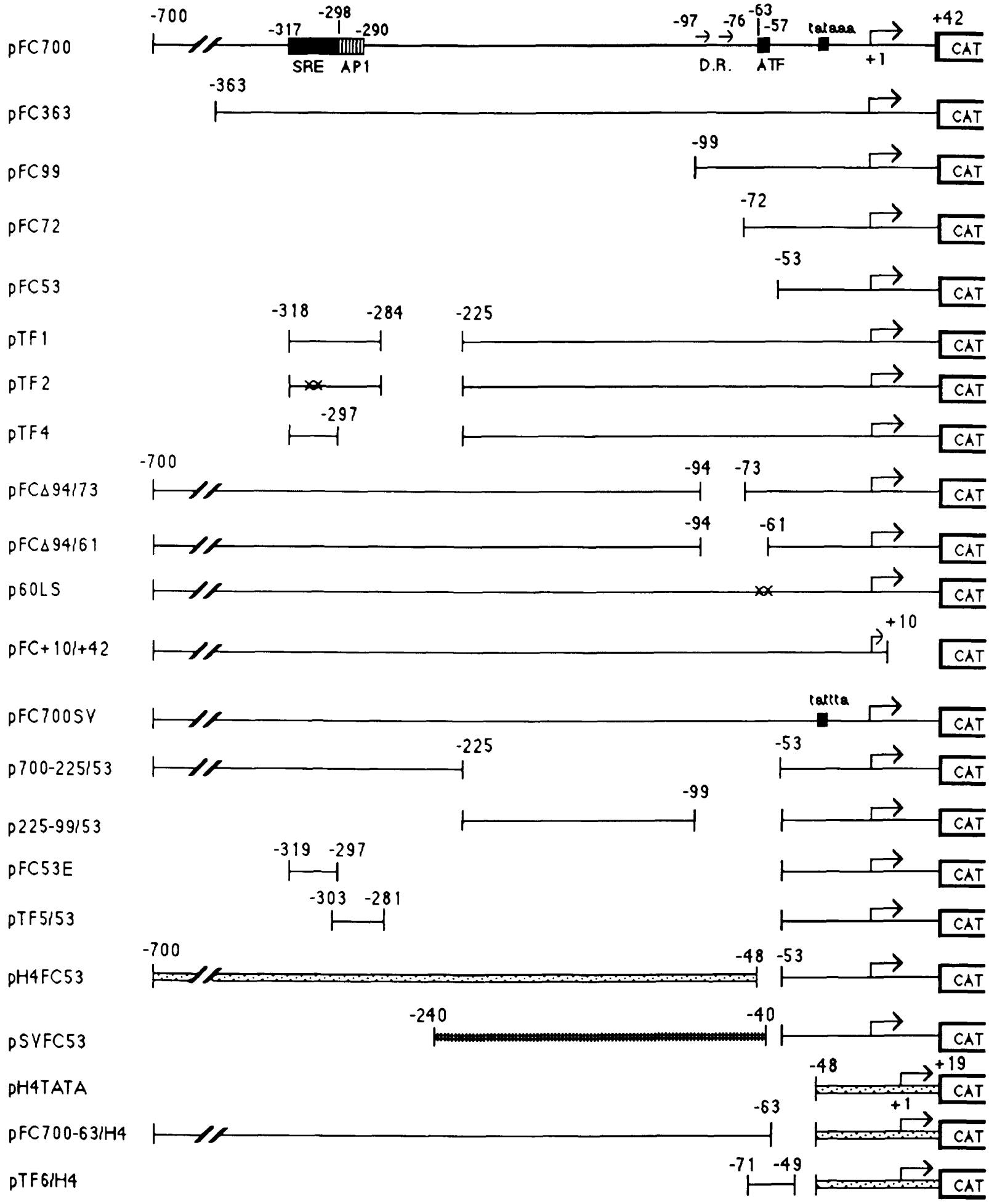

Figure 2. Diagram of the fosCAT constructs used in this study. As described in the text, the fosCAT genes are hybrid constructs with parts of the human c-fos gene, extending from variable $5^{\prime}$ sites to position +42 , fused to the coding region of the bacterial gene for chloramphenicol acetyltransferase (CAT) (Fisch et al. 1987). At the top of the figure, five relevant sequence elements in the c-fos promoter are shown schematically. The SRE and ATF sequences and the TATA box are symbolized by dark boxes. The AP1 binding site is symbolized by a lined box. The direct repeats (D.R.) are indicated by arrows. The point mutations in pTF2 (in the SRE) and the linker scanning mutation in p60LS (in the ATF site) are indicated by $\times$. The sequence of the point mutation in pFC700SV is detailed. Sequence from the human histone H4A gene is symbolized by dotted rectangles. Sequence from the SV40 early promoter/enhancer is symbolized by a hatched rectangle. 


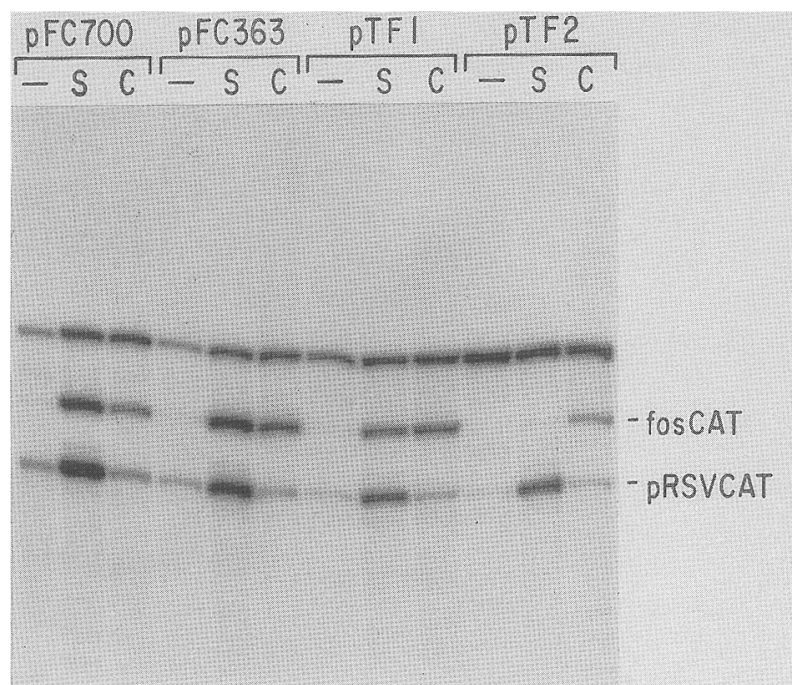

Figure 3. Induction of fosCAT fusion genes in NIH-3T3 cells by cAMP is independent of the SRE. NIH-3T3 cells were transfected with the indicated fosCAT constructs, diagramed in Fig. 2. pRSVCAT was cotransfected as an internal control for transfection efficiency. After transfection (16 hr after) the cells were incubated for an additional $24 \mathrm{hr}$ in medium containing $0.5 \%$ bovine calf serum, as described in the text. Total cellular RNA was prepared either before (lanes - ) or $30 \mathrm{~min}$ after stimulation with $10 \%$ bovine calf serum (lanes S) or $1 \mathrm{~mm} 8 \mathrm{Br}$-cAMP (lanes $\mathrm{Cl}$ and analyzed by S1 mapping; $20 \mu \mathrm{g}$ of RNA was used per reaction. Self-hybridized Sl probe, fosCAT, and pRSVCAT-specific protected fragments are indicated by arrows.

fosCAT constructs including and lacking a functional SRE (all described previously; Fisch et al. 1987) were introduced transiently into NIH-3T3 cells and induced with $10 \%$ serum or with 8 -Br cAMP. Plasmid pFC700; including 700 base pairs of promoter sequence upstream of the cap site, pFC363, including 363 base pairs of upstream sequence and pTF1, which contains a 40-basepair oligonucleotide spanning region -318 to -284 in the c-fos promoter fused at position -225 to the fosCAT fusion construct, were all induced by serum (about tenfold) and by 8-Br cAMP (about sevenfold; these data and all subsequent data shown were quantitated by densitometric scanning, as described in Materials and methods). Notably, the reference plasmid pRSVCAT also was induced strongly by serum, a finding that was reproduced in other experiments (data not shown). Plasmid pTF2 was much less responsive to serum than the other constructs, but was still strongly induced by cAMP. This plasmid is similar to pTF1, except that it contains four point mutations in the SRE, so that, unlike PTF1, it does not compete for binding of the serum response factor (SRF) to the SRE in gel mobility shift assays (R. Prywes and R.G. Roeder, unpubl.).

Other sequence elements in the $c$-fos promoter are not individually required for induction by $C A M P$

Since our initial experiments indicated that the SRE was not required for CAMP induction, we investigated whether other sequence elements in the human c-fos promoter might be required for this response. In particular, two sequence elements in the c-fos enhancer/promoter bear strong homology to previously described cAMP-responsive sequence elements in other genes (Comb et al. 1986; Montminy et al. 1986, 1987) and thus seemed likely candidates for the role of cAMP regulatory element in the c-fos promoter (Table 1). One of these sequence elements, between positions -63 to -57 in the human c-fos promoter, has been shown previously to be important for basal expression of both human and murine c-fos (Gilman et al. 1986; Fisch et al. 1987). It is identical in sequence to adenovirus promoter elements that bind the so-called activating transcription factor (ATF; Fisch et al. 1987; Lee and Green 1987; Lin and Green 1988), which is important for expression of a variety of adenovirus early genes (SivaRaman et al. 1986; Lee and Green 1987; Hardy and Shenk 1988; Hurst and Jones 1988; Lin and Green 1988). The c-fos element at -60 is also highly homologous to the cAMP-responsive element (CRE) in the human somatostatin promoter (Table 1). The other element, located between positions -298 to -287 relative to the mRNA cap site, is identical in 8 of 9 base pairs to the AP1 consensus binding site and in 11 of 12 base pairs to the AP1-like sequence element responsible for cAMP and TPA-induction of the human preproenkephalin gene (Table 1; Comb et al.

Table 1. Homologies of three different sequences in the fos promoter

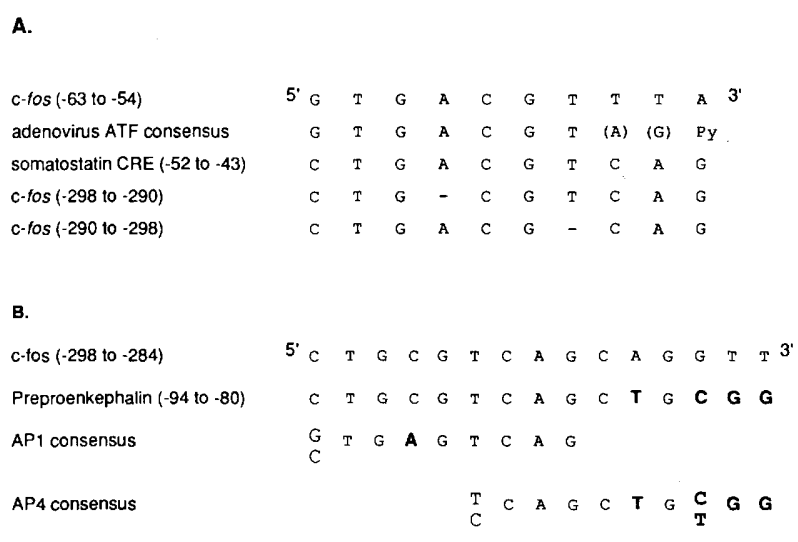

(A) Homology of the c-fos sequence at -63 and at -298 to adenovirus ATF binding sites and to the CRE. c-fos sequences from -63 to -54 and from -298 to -290 is compared to the rat somatostatin cAMP response element (CRE; Montminy et al. 1987) and to an adenovirus ATF consensus sequence derived from ATF binding sites in the adenovirus E1A, E2A, E3, and E4 early promoters (Lin and Green 1988). Parentheses surround bases that are less strongly conserved. The c-fos sequence from -298 to -290 is shown in both orientations.

(B) Homology of the c-fos sequence at -298 to the APl and AP4 consensus binding sites and to a cAMP-responsive sequence in the human preproenkephalin gene. c-fos sequence from -298 to -284 is compared to the consensus binding sites for AP1 (Angel et al. 1987; Lee et al. 1987) and AP4 (Mermod et al. 1988) and to the cAMP/TPA-responsive sequence element in the human preproenkephalin (PPE) gene (Comb et al. 1986). Bases that differ from the c-fos sequence are in boldface. 
1986; Angel et al. 1987; Fisch et al. 1987; Lee et al. 1987). Included within the c-fos sequence homologous to the AP1 consensus is another sequence that is homologous to the ATF site located at -63 in c-fos $15 / 7$ base pairs) and to the CRE element in the somatostatin promoter (6/7 base pair match). Finally, overlapping the AP1 homology is a sequence with a weaker homology to the binding site for transcription factor AP4 /6/10 base pairs; Mermod et al. 1988). Thus, the region from -298 to -284 may bind potentially at least two proteins /see Discussion). However, for simplicity, we shall refer to this region as the 'API consensus homology.' We also wanted to determine whether the region spanning -225 to -99 and the region spanning the direct repeat sequence element $(-97$ to -76$)$ were responsive to cAMP. The direct repeat sequence is involved in the basal level of expression of human c-fos, but has no effect on the level of induction by EGF or TPA in HeLa cells (Fisch et al. 1987).

Figure 4 shows the results of an experiment in which five fosCAT fusion genes which lacked the candidate regulatory elements singly or in combination were transfected transiently into NIH-3T3 cells and exposed to $8-\mathrm{Br}$ cAMP for $30 \mathrm{~min}$. These constructs are diagramed in Figure 2. Plasmid pTF4 contains a 27-basepair oligonucleotide spanning the SRE (positions -319 to -297 ) fused at position -225 to the fosCAT fusion construct. This construct therefore lacks the APl consensus homology (although the SRE and sequence downstream of -225 is intact). Plasmid pFC99 is a $5^{\prime}$ deletion mutant that lacks sequences upstream of position -99 . Plasmid pFC $\Delta 94 / 73$ is an internal deletion mutant of plasmid pFC700 that is missing sequences between positions -94 and -72 , and thus the direct repeat sequences. Similarly, pFC $\Delta 94 / 61$ is missing sequences be-

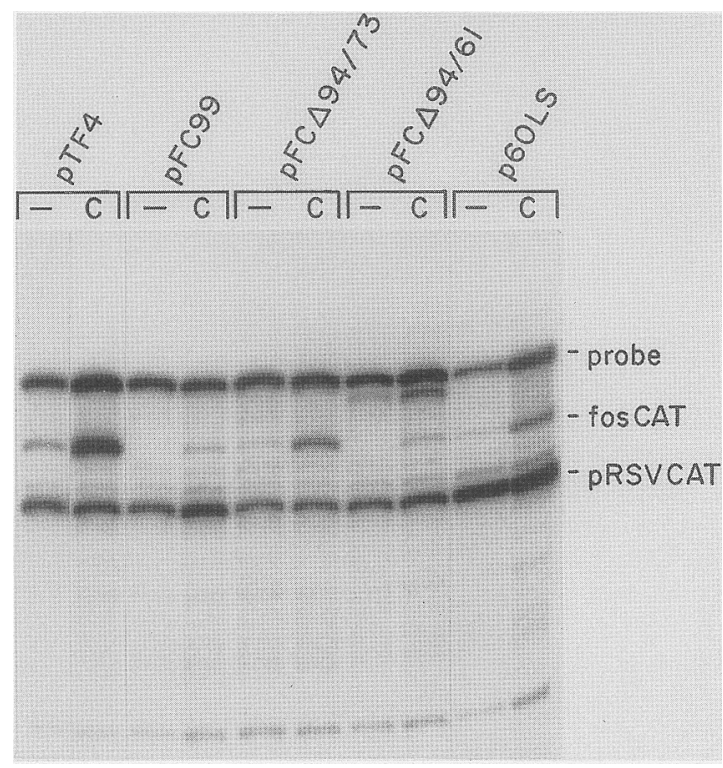

Figure 4. Induction by cAMP of fosCAT genes lacking different promoter regions. NIH-3T3 cells were transfected with the indicated fosCAT constructs, treated with 8-Br cAMP, and analyzed for expression as described in the legend to Fig. 3. tween positions -94 and -61 ; the deleted region includes both the direct repeat sequences and the ATF consensus homology. We have shown previously that pF $\Delta 94 / 61$ lacks a functional ATF site; this mutant showed diminished basal expression in vivo and did not compete in gel mobility shift assays for binding of a nuclear protein to the ATF consensus homology (Fisch et al. 1987). ATF sites in adenovirus E4 and Ela genes and in the c-fos gene competed equally well for binding of this nuclear factor, which was therefore presumably ATF (Fisch et al. 1987). Finally, p60LS is a linker scanning mutant in which a $B g I I I$ linker disrupts the first two base pairs of the ATF homology. Like pFC $\Delta 94 / 61$, this mutant showed diminished basal expression upon transfection and did not compete with the intact sequence in gel mobility shift assays for the binding of ATF (data not shown).

The five constructs described above were all induced about eightfold by cAMP, a level of induction comparable to that which has been observed for the 'wild-type' gene pFC700 (the autoradiograph shown in Fig. 4, as well as darker and lighter exposures of the same experiment, was quantitated by densitometric scanning). Furthermore, pFC $\Delta 94 / 30$, another internal deletion mutant lacking all sequences between -94 and the TATA box (at position -30) was also fully inducible by cAMP (data not shown). Thus, no one sequence element upstream of -30 was alone necessary for cAMP induction of $\mathrm{c}-$ fos expression.

Notably, in Figure 4 the expression of pFC99 was quite low relative to that of pTF4. This diminished expression may have reflected the juxtaposition of transcriptionally inhibitory sequences in the pUC19 vector to the proximal portion of the c-fos promoter in addition to deletion of specific protein-binding sites upstream of -99 . In fact, removal of sequence upstream of $-225 \mathrm{had}$ no effect on fosCAT expression, and internal deletion of sequences between -225 and -99 caused only a twofold reduction in expression in NIH-3T3 cells (data not shown). In HeLa cells, the elimination of sequences between -225 and -99 had no effect on basal expression of c-fos (Fisch et al. 1987).

\section{Sequences downstream of -53 are not required for induction of $c$-fos by $c A M P$}

In light of the results thus far described, two possible hypotheses explaining the mechanism of the cAMP response remained to be tested. The first was that the promoter contained multiple elements that could independently mediate the full response to cAMP. The second was that sequence elements between -30 to +42 in the c-fos promoter mediated the cAMP response, as no one sequence element upstream of -30 could be shown to be necessary for cAMP induction. The major recognized sequence element in the -30 to +42 region is the TATA box.

We conducted a series of experiments to test the second hypothesis. First, we examined the effect of sequence between the TATA box and +42 by constructing 


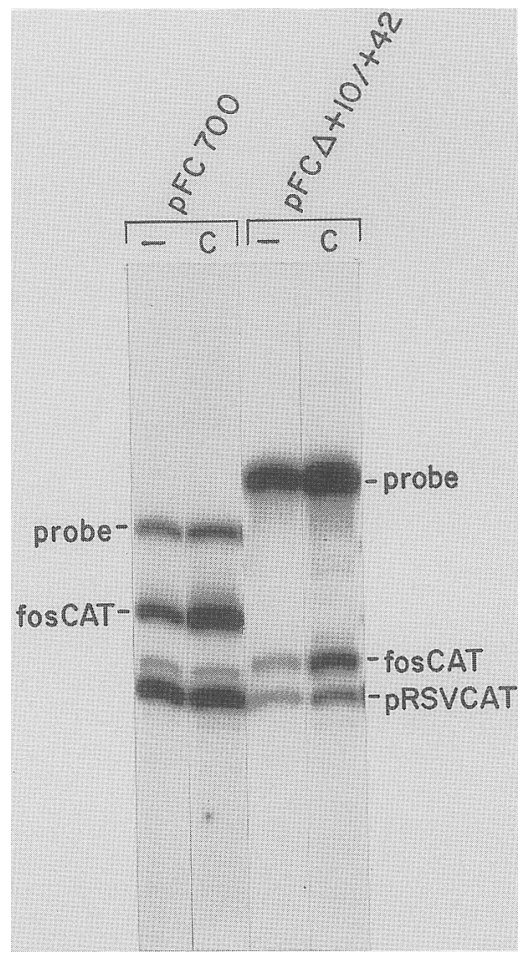

Figure 5. A fosCAT construct lacking sequence between +10 and +42 is induced by cAMP. Cells were transfected with $\mathrm{pFC} 700$ and $\mathrm{pFC} \Delta+10 /+42$, an internal deletion mutant of pFC700 lacking sequence between +10 to +42 in c-fos. Cells were treated with $8-\mathrm{Br}$ cAMP and analzyed for expression as described in the legend to Fig. 3. For analysis of transcripts from $\mathrm{pFC} \Delta+10 /+42$, an approximately 490 -bp probe was prepared from that plasmid. The probe included 250 bp of CAT coding sequence and c-fos sequence from -225 to +10 . Because transcripts from pFC $\Delta+10 /+42$ include only 10 bp of c-fos $5^{t}$ untranslated sequence, the specific protected fragment is only 10 bp larger than the fragment protected by pRSVCAT transcripts.

an internal deletion mutant of pFC700 lacking sequences between position +10 and +42 relative to the mRNA cap site $(\mathrm{pFC} \Delta+10 /+42$; described in Materials and methods). As shown in Figure 5, this construct was inducible by cAMP. Although the level of induction of $\mathrm{pFC} \Delta+10 /+42$ was low in this experiment, it was comparable to that of the control plasmid pFC700 (fourfold induction, as determined by densitometric scanning, with normalization to the pRSVCAT control). In another experiment, $\mathrm{pFC} \Delta+10 /+42$ was induced fivefold. Similarly, a construct deleted in sequence between positions -10 and +42 remained as inducible as $\mathrm{pFC} 700$ by cAMP (data not shown). These results showed that sequences between position -10 and +42 were not required for the cAMP response, although it is possible that sequence elements located in this region might function interchangeably with elements located upstream of the TATA box. We consider the latter possibility unlikely, as a $5^{\prime}$ fosCAT deletion mutant lacking all sequence upstream of -53 (pFC53) was apparently not inducible by cAMP (Fig. 8A, lanes 7,8 ) and showed neither basal nor cAMP-induced expression even on very long autoradiographic exposure (data not shown). The latter result also suggested that the TATA element and associated factors alone were not sufficient to convey cAMP-inducibility to the c-fos promoter. However, this result did not definitively disqualify the TATA motif as a potential cAMP regulatory element, as the possibility remained that it might require the presence of upstream elements to effect a basal level of activity that could be further elevated by cAMP.

To test this possibility, upstream elements from other promoters not responsive to cAMP were fused to position -53 of the fosCAT fusion gene. The first /creating pH4FC53) was a region $(-700$ to -48$)$ from the human histone $\mathrm{H} 4 \mathrm{~A}$ gene that contains binding sites for at least two known transcription factors, H4TF1 and SP1, and contributes to expression of the H4A gene (Zhong et al. 1983; Dailey et al. 1986; S.M. Hanly et al., in prep.). The second (creating pSVFC53) was a region $(-270$ to -37$)$ containing the promoter/enhancer region of SV40. Figure 6A shows the analysis of these constructs following transfection into NIH-3T3 cells. pH4FC53 was expressed at a low level which was not induced by $8-\mathrm{Br}$ cAMP, while pSVFC53 was expressed at a higher level which was also unaffected by 8 -Br cAMP (pSVFC53 was induced about twofold by serum; data not shown). In the same experiment, pFC700 was induced about fivefold by cAMP, as determined by densitometric scanning of another appropriately exposed autoradiogram. Thus, in the context of these chimeric promoters, the c-fos TATA box did not convey cAMP-inducible expression. The fact that $\mathrm{pH} 4 \mathrm{FC} 53$ was not induced by cAMP also ruled out the possibility that juxtaposition of inhibitory vector sequences to the proximal region of the c-fos promoter inhibited cAMP induction of the fosCAT construct pFC53.

Another reason why the c-fos TATA box seemed unlikely to mediate the CAMP response was that not all genes with TATA boxes were induced in our transient assays. For example, pRSVCAT was not induced by cAMP. However, there is precendent for a TATA boxmediated transcriptional induction that works only through a particular TATA sequence. In cotransfection experiments, adenovirus Ela gene products induced expression of genes such as human $h s p 70$, containing the sequence TATAAA, but not of the SV40 early promoter, containing the sequence TATTTA (Simon et al. 1988). In fact, the sequence of the c-fos TATA box is TATAAA and that of the RSV TATA box is TATTTA. In view of these results, we tested whether the sequence TATAAA was critical for the cAMP response of the c-fos gene by changing the sequence to TATTTA in the pFC700 plasmid. Figure 6B shows that the TATA mutant pFC700SV was induced as well as pFC700 by cAMP (fivefold for each, as determined by densitometry of appropriate exposures), although the basal level of its expression was much lower than that of pFC700. Thus, the sequence TATAAA is not required for induction of $c$-fos by cAMP. In addition, the promoter of the human histone gene $\mathrm{H} 4 \mathrm{~A}$, which includes a TATA sequence identical to that of c-fos, was not responsive to cAMP. Figure 


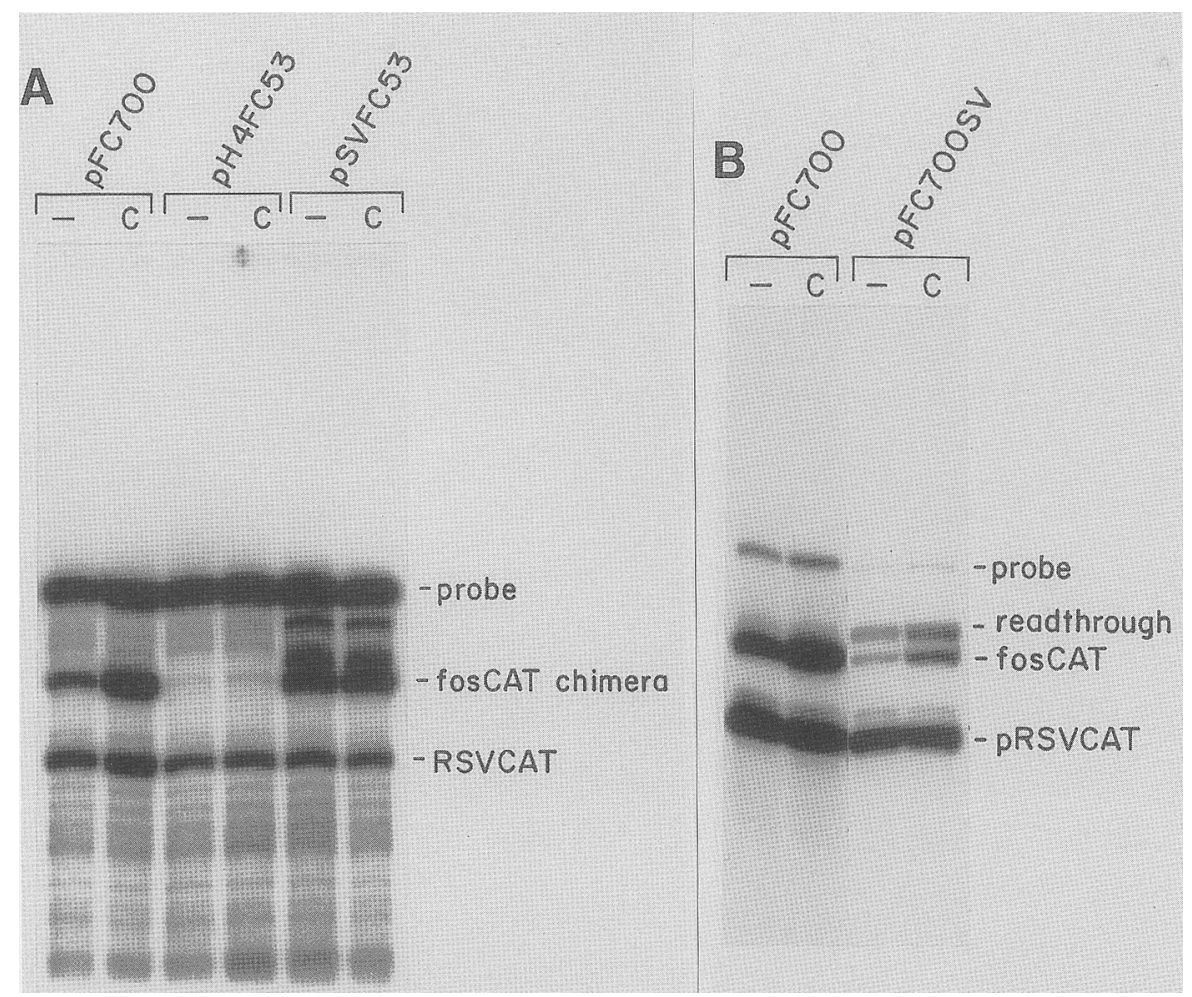

Figure 6. $|A|$ Induction of chimeric human histone H4/fosCAT and SV40/fosCAT constructs by cAMP. NIH-3T3 cells were transfected with chimeric constructs containing sequence from the promoter of the human histone H4A gene (sequence from -700 to -48 of phuH4A) or from the SV40 early promoter/enhancer ( -270 to -37 ) fused to position -53 of the fosCAT construct (called pH4FC53 and pSVFC53, respectively). Cells were treated with 8-Br cAMP and analyzed for expression as described in the legend to Fig. 3. $(B)$ Induction of a fosCAT TATA box point mutant by cAMP. Cells were transfected with pFC700 and with pFC700SV, a point mutant containing two single base pair changes in the TATA box (TATAAA changed to TATTTA). pRSVCAT was cotransfected as an internal control. After transfection $(28 \mathrm{hr}$ ), the transfected cells were left untreated (lanes - ) or exposed to a 30 min treatment with 8 Br-cAMP (lanes C), after which total cellular RNA was harvested (the cells were not incubated in low serum medium in this experiment, as discussed in the text). An aliquot of RNA $(50 \mu \mathrm{g})$ was used per S1 reaction. The band just above the specific pFC700SV fragment corresponds to transcripts initiated upstream in the pUC19 vector. It is distinguishable by size from the probe prepared from pFC700 because of the discontinuity in the TATA region.

7A (lanes 3,4) shows that a fusion construct composed of a segment of the H4A gene from -700 to +19 linked to the coding sequence of CAT (p21303CAT; S.M. Hanly et al., in prep.) was not induced by cAMP. Therefore, if the cAMP induction of $c$-fos is mediated via a TATA recognition factor, this induction must depend on sequence differences in the flanking regions of the c-fos and H4 TATA boxes. We consider this possibility unlikely.

$c$-fos upstream sequences convey cAMP inducibility to an unresponsive promoter

Our experiments indicated that sequences in the c-fos promoter upstream of the TATA box mediate transcriptional induction by cAMP. To substantiate this hypothesis, we tested whether the cAMP response could be transferred to an unresponsive promoter by joining $\mathrm{c}$-fos upstream sequences to position -48 of the H4-CAT fusion gene described above ( 2 21303CAT). We created two complementary constructs: one, pFC700-63/H4, in which sequences from -700 to -63 from c-fos were li- gated to position -48 of the H4-CAT construct; and a second, pTF6/H4, in which a 28-base-pair oligonucleotide spanning positions -71 to -49 in c-fos (including the ATF binding site) were ligated to position -48 of the H4-CAT construct. These constructs were analyzed in NIH-3T3 cells, alongside p21303CAT and pH4TATA, a 5 ' deletion mutant of the H4-CAT fusion construct lacking $\mathrm{H} 4$ promoter sequence upstream of position -48 . The response of these constructs to a $30-\mathrm{min}$ treatment with 8-Br cAMP is shown in Figure 7 /Fig. 7B is a lighter exposure of the same experiment shown in Fig. 7A). Neither p21303-CAT nor pH4TATA were induced by cAMP, but when normalized to the pRSVCAT internal control, pFC700-63/H4 was induced about threefold and $\mathrm{pTF} 6 / \mathrm{H} 4$ was induced about fivefold. (Although the induction of pTF6/H4 as seen in Fig. 7A, lane 10 seems quite large, pRSVCAT also was expressed considerably better in the same cAMP-treated cells, as seen in Fig. 7b, lane 10. When normalized to the 'induction' of pRSVCAT, the induction of pTF6/H4 was fivefold.) In a parallel transfection, $\mathrm{pFC700}$ was induced about fivefold (Fig. 7A,B, lanes 1, 2). Thus the ATF site and c-fos se- 


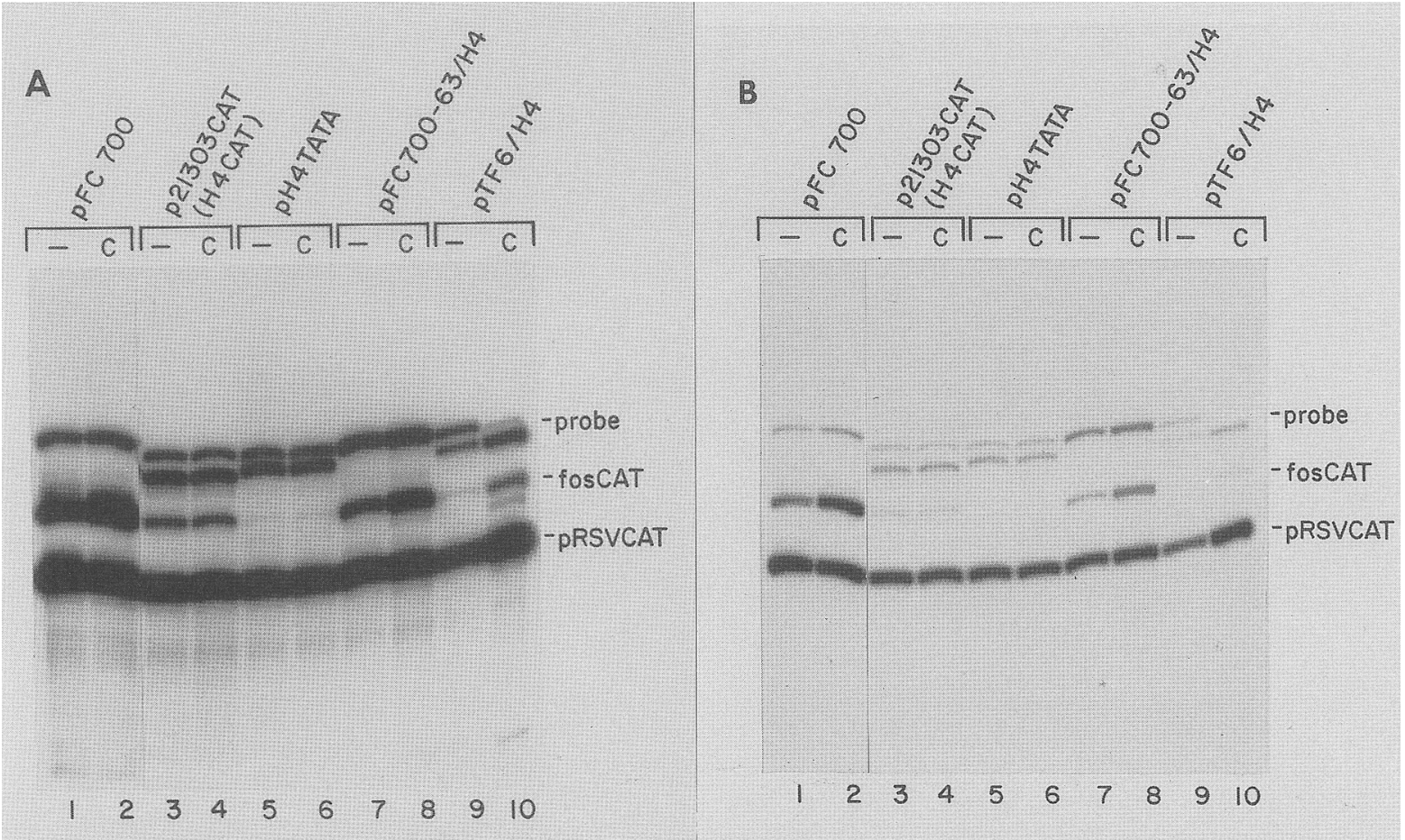

Figure 7. (A) Induction of human histone H4-CAT constructs and of fos/H4-CAT chimeric genes by cAMP. Cells were transfected with human histone H4A-CAT fusion genes containing 700 base pairs (p21303-CAT) or 48 base pairs (pH4TATA) of 5 ' flanking sequence from the huH4A promoter and with c-fos/H4CAT chimeric genes including c-fos $5^{\prime}$ flanking sequence from -700 to -63 (p700-63/H4) or from -71 to -48 (pTF6/H4) fused to position -48 of the H4A-CAT fusion gene. Cells were treated with 8-Br cAMP and analyzed for expression as described in Fig. $6 \mathrm{~B}$. The probe was an $\sim 360$-base-pair fragment prepared from p $700-63 / \mathrm{H} 4$ which included 250 base pairs of CAT coding sequence, H4A $5^{\prime}$ sequence from -48 to +19 and c-fos $5^{\prime}$ flanking sequence from -100 to -63 . Rehybridized probe and specific protected fragments are indicated. Bands that migrated between the specific protected H4-CAT fragments and the probe correspond to transcripts initiated upstream in the pUC18 or pUC19 vectors. They are distinguishable from the probe because of the discontinuity starting at -48 in H4A. (B) A lighter exposure of the same experiment shown in Fig. $7 \mathrm{~A}$, so that the level of induction of more strongly expressed constructs (i.e., pFC700 and pRSVCAT) might be assessed.

quence upstream of -63 can convey cAMP responsiveness to a normally refractory promoter; however, the c-fos sequence within the -700 to -63 region seem to convey a somewhat greater induction in the context of the c-fos promoter.

\section{Multiple individual elements in the c-fos promoter can mediate induction by cAMP}

The lack of a requirement for any specific sequence element for the cAMP response of c-fos led us to the hypothesis that multiple elements might be able to act independently. The induction of TF $6 / \mathrm{H} 4$ by cAMP demonstrated that the ATF binding site in the c-fos promoter is a cAMP-responsive sequence element. To test the cAMP responsiveness of this and other individual sequence elements in the context of the c-fos promoter, we fused various fragments or oligonucleotides comprising distinct regions in the c-fos promoter to position -53 of pFC53. Surprisingly, we found that at least four elements in the c-fos promoter could mediate induction by cAMP (Fig. 8). The plasmids we tested were p700-225/ 53 , containing sequences from -700 to -225 ligated to position -53 (including both the SRE and the AP1 consensus homology|; pFC53E, containing a 27-base-pair oligonucleotide including the SRE, but not the AP1 con- sensus sequence, ligated to position -53 of pFC53; $\mathrm{p} 225-99 / 53$, containing sequences from -225 to -99 fused to position -53; and $\mathrm{pFC72}$, a $5^{\prime}$ deletion mutation lacking sequence upstream of -72 but retaining the ATF site at -63 to -57 (see Fig. 2 for diagrams). In transient transfection assays, all of these constructs were induced about fivefold by cAMP / while the induction of pFC700 in Fig. 8 appears low, in shorter exposures the induction was quantitated to be fivefold). In addition, pTF5/53, which contains a 28-base-pair oligonucleotide spanning positions -303 to -281 in c-fos (including the AP1 consensus but not the SRE sequence) ligated to position -53 of pFC53, was induced 10 -fold by cAMP, as opposed to the 5 -fold induction of pFC700 (Fig. 8B), a finding that was consistent in three repeated experiments. Notably, the 27-base-pair oligonucleotide used to create $\mathrm{pFC53 \textrm {E }}$ competed for binding to serum response factor (SRF) in gel mobility shift assays (R. Prywes and R.F. Roeder, unpubl.); similary, the 28-base-pair oligonucleotide used to create pTF5/53 competes with the SV40 AP1 site for binding to a protein in HeLa nuclear extracts (E. Gagne and R.G. Roeder, unpubl.). Thus, at least four sequence segments fused to -53 of the $c$-fos promoter can mediate the cAMP response: $(1)-317$ to -198 , spanning the SRE; (2) -303 to -281 , spanning the AP1 consensus homology land also the ATF ho- 
Fisch et al.

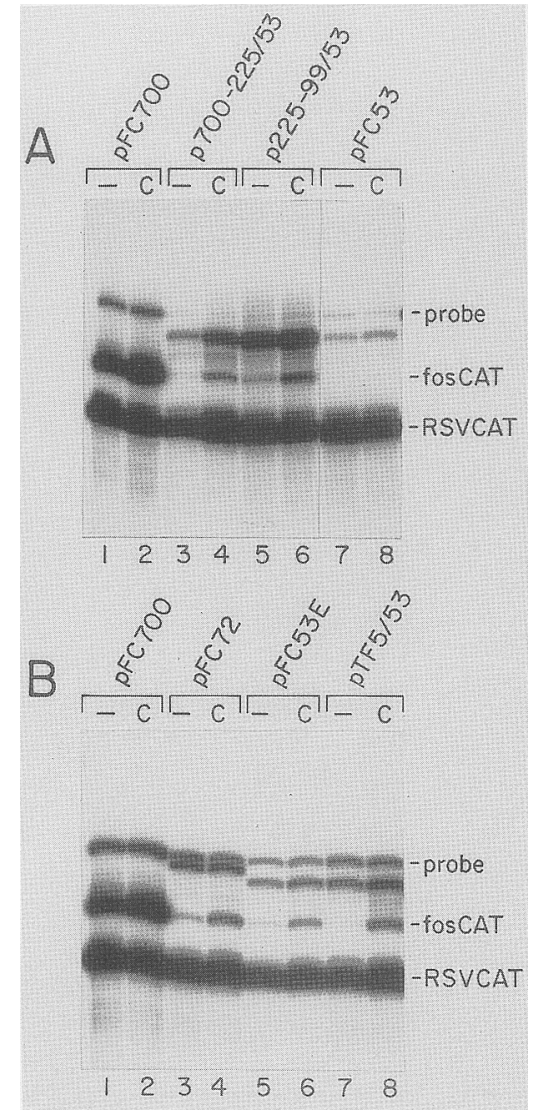

Figure 8. (A) Sequence between positions -700 to -225 and -225 to -99 in c-fos convey cAMP induction to pFC53. Cells were transfected with the indicated constructs diagramed in Fig. 2. Cells were treated with 8-Br cAMP and analyzed for expression as described in the legend to Fig. 6B. Bands migrating between the probe and specific protected bands correspond to transcripts initiated upstream in the pUC19 vector that are distinguishable from the probe (derived from pFC700) because of the discontinuity at position -53 . $(B)$ Inducibility by cAMP is conveyed by three distinct sequence elements in the c-fos promoter. Cells were transcribed with the indicated constructs, treated with $8-\mathrm{Br}$ cAMP and analyzed as described above. pFC72 contains the ATF binding site at -63 to -57 . pFC53E contains a 28-base-pair oligonucleotide spanning the SRE and pTF5/53 contains a 28-base-pair oligonucleotide spanning the APl consensus sequence.

mology at -295$) ;(3)-225$ to -99 ; and $(4)-72$ to -54 , spanning the ATF/CRE homology.

\section{Discussion}

The object of our study was to identify the sequence elements responsible for induction of c-fos by cAMP, and two important conclusions ensued. First, our results have distinguished induction by cAMP from induction by serum, EGF, and TPA. Thus, deletion of the SRE, an element required by the latter agents (Treisman 1985; Gilman et al. 1986; Fisch et al. 1987; Greenberg et al. 1987) had no effect on the cAMP response. These findings are in agreement with a recent report by Gilman (1988), who found that induction of murine c-fos in BALB/c-3T3 cells by cAMP was independent of the SRE, but that induction by TPA and c-sis protein (B-chain homodimers of platelet-derived growth factor) was SRE dependent. Second, and somewhat surprisingly, we found that multiple sequence elements in the c-fos promoter were involved in inducibility by cAMP and, furthermore, that these sequence elements could function independently. Thus, deletion of specific sequence elements from the promoter either singly or in combination did not impair inducibility of c-fos by cAMP. Of special note was the observation that cAMP induction was not impaired by internal deletion or point mutation of the - $60 \mathrm{ATF} / \mathrm{CRE}$ homology, known to be important for cAMP induction of other genes, as described above. At least three other sequence elements (described below) could substitute for the ATF/CRE site to maintain cAMP induction, and these elements could function in the context of a relatively intact c-fos promoter structure. Only removal of all sequences upstream of position -53 rendered the fosCAT fusion gene unresponsive to treatment with cAMP.

When tested by fusion to position -53 of the cAMPunresponsive plasmid pFC53, at least four distinct sequence elements were found to mediate independently the cAMP response as effectively (fivefold) as the wildtype gene pFC700 in parallel transfections. These included: (1) a 28-base-pair oligonucleotide reconstituting the SRE; (2) the ATF homology at its normal location at -63 to -57 ; (3) an element that spanned the AP1 consensus homology $(-303$ to -281$)$ and which was induced reproducibly to a greater extent (10-fold) than the wild-type gene pFC700; and (4) the region between positions -225 to -99 . The first three elements were well defined to within about 20 base pairs and correspond to previously known sequence elements, but we have not identified precisely the specific sequence element/s) within the region from -225 to -99 that mediate $(\mathrm{s})$ the cAMP effect. However, we have noted that this region includes several stretches of sequence that are homologous to the consensus binding site for the transcription factor AP2. This site can mediate transcriptional induction of the human metallothionein IIA (hMT-IIA) gene by cAMP and TPA (Imagawa et al. 1987). The consensus binding site for AP2 is quite degenerate, however, and in the absence of binding studies with purified protein it is hard to evaluate the significance of sequence homologies to this consensus.

Thus, while the ATF/CRE element can mediate cAMP inducibility of the c-fos gene, it is not stringently required for induction. Other sequence elements can mediate cAMP induction, either individually or in the context of a CRE-depleted, but otherwise relatively intact, c.fos promoter. Given this redundancy in functional cAMP-response elements, it is hard to assess by deletion or point mutation the individual contributions of given sequence elements to the cAMP response in the context of the fully intact promoter. We discuss below cAMP induction models by which one or all of the cAMP-re- 
ponsive elements might convey induction in the intact promoter.

What is striking about the present results is that the degree of induction by cAMP was the same whether all or only a subset of the cAMP-responsive elements were present in the promoter. Thus, fosCAT constructs lacking one or more of the cAMP-responsive promoter elements were induced just as much (five- to eightfold in different experiments) as the construct containing all of the elements. Because of the consistent five- to eightfold response to cAMP of the wild-type promoter and the various fosCAT deletion mutants, we conclude that the respective transcription factors that function through the cAMP-responsive sequence elements do not interact synergistically. If they did, the induction with all of them present should be greater than the induction with only a subset present. At the same time, it important to note that both the basal levels of activity and the absolute levels of activity in the presence of cAMP differed significantly for the different constructs.

Several models are consistent with the cAMP-responsive sequence elements in the c-fos promoter contributing independently, but not synergistically, to expression. One model is that the four cAMP-responsive elements contribute additively to basal expression and that cAMP induces the contribution to each element to the same degree (around fivefold; for example, by increasing the transcriptional activity of the protein factors that function through the various cAMP-responsive sequence elements). Thus, the total promoter activity in the induced state would be stimulated in proportion to the activity of the individual promoter elements, e.g., to five times the total basal promoter activity. A second model is that, although all the elements are potentially responsive to cAMP, only one is used to activate transcription. Thus, a particular sequence element might be preferred, possibly because of its location in the promoter, but in its absence induction could still be mediated by another cAMP-responsive element. Similarly, in different cell types, different sequence elements might be used for the cAMP induction, depending on transcription factor abundance. Further experimentation will be necessary to distinguish between the different models that explain the constant magnitude of the cAMP induction.

Although almost all the fosCAT constructs tested exhibited the same magnitude (around fivefold) of induction by cAMP, one (pTF5/53) was reproducibly induced at least tenfold in different experiments. The latter construct contains three different sequence homologies (to consensus binding sites for AP1, ATF, and AP4, as discussed above) and there are two possible explanations for its greater inducibility. One is that the factor which functions through the TF5 oligonucleotide sequence $(-303$ to -281$)$ is induced to a greater extent than the other cAMP-responsive factors. This would imply that induction through pTF5 is not mediated by the ATF protein. Alternatively, ATF may be working through the TF5 sequence, but, for some reason, the basal level of pTF5/53 might be artificially low compared to that of
pFC72 (which includes the ATF site at -63 ). This could occur if, for example, other protein factors competed effectively for binding in the uninduced state to the TF5 sequence. It has been demonstrated that APl can bind to the sequence between -303 to -281 (Piette and Yaniv 1987; E. Gagne and R.G. Roeder, unpubl.). ATF can also bind to this region, although with substantially lower affinity (5- to 10-fold lower) than to the ATF site at -63 in c-fos (E. Bernadette et al., unpubl.). A finer mutational analysis accompanied by both binding and functional studies will be necessary to determine which factor mediates cAMP induction through this site. It is interesting to note that multimers of the AP1 site fused to the $\beta$ globin promoter conferred induction by TPA but not cAMP in HeLa tk- cells (Imagawa et al. 1987).

While analyzing the upstream cAMP-responsive elements, we also made several observations that argue against the possibility that cAMP induction might be mediated through the TATA element and associated factors. First, other TATA-containing genes were not induced by NIH-3T3 cells by cAMP. Second, conversion of the c-fos sequence (TATAAA) to that of the cAMP-uninducible RSV and SV40 promoters (TATTTA) did not impair function of the fosCAT construct by cAMP. Third, sequences located upstream of the TATA box in the c-fos promoter were able to confer cAMP-inducibility to the cAMP-unresponsive histone H4A promoter. Fourth, neither pFC53 nor chimeric constructs consisting of upstream sequence from other promoters /the human histone $\mathrm{H} 4 \mathrm{~A}$ promoter or the SV40 promoter/enhancer) fused to position -53 of pFC53 were inducible by cAMP. (Even though the SV40 promoter/enhancer contains binding sites for both AP1 and AP2, their effect may not be apparent in the context of the other sequence elements in the SV40 promoter.)

Three different cAMP-responsive sequence elements have been identified previously in the promoters of a variety of genes. A number of cAMP-inducible genes, including the rat somatostatin and the human vasoactive intestinal peptide (VIP) genes, are induced through the so-called CRE (Montminy et al. 1986). The palindrome $5^{\prime}$-CTGACGTCAG- ${ }^{\prime}$ ' is the hallmark of the CRE; it is contained within a region in the rat somatostatin gene necessary for induction of that gene by cAMP (Montminy et al. 1986), although the palindrome by itself was not sufficient to convey cAMP-inducibility to an unresponsive promoter. However, a 17-base-pair region from the human VIP gene, including the 5-base-pair motif 5'CGTCA-3' and an inverted copy of this motif, was sufficient to convey cAMP inducibility to a normally unresponsive gene (Tsukada et al. 1987). The second cAMPresponsive sequence element is found in the promoter $(-100$ to -71$)$ of the human preproenkephalin gene (Comb et al. 1986). This sequence element contains a sequence motif that is highly homologous to the AP1 consensus binding site (8/9 base pairs; Angel et al. 1987; Lee et al. 1987). Overlapping the AP1 consensus homology in the preproenkephalin promoter is a sequence homologous to the consensus binding site for transcription factor AP4 (9/10 base pairs; Mermod et al. 1988). 
The third known cAMP-responsive sequence element is the binding site for the transcription factor AP2, which is found in the cAMP-responsive human metallothionein IIa gene. Only one of the three different types of cAMP-responsive sequence elements is found in the promoter of any one of these cAMP-inducible genes. Thus, the c-fos promoter/enhancer is rather unusual in that it contains multiple sequence elements that are responsive to cAMP. However, it is similar to the other cAMP-responsive genes in that three of four cAMP-responsive elements that we have identified in the c-fos promoter are homologous to the single cAMP-responsive elements contained in the other cAMP-responsive promoters. Moreover, induction of c-fos by cAMP is not the only reported case of an induction that occurs through multiple sequence elements. Multiple elements in the SV40 promoter are responsive to induction by TPA /Chiu et al. 1987), and multimers of the SV40 B, C, and P elements cloned downstream of the $\beta$-globin promoter conferred TPA inducibility to that promoter in HepG2 cells and to a lesser extent in HeLa tk- cells.

In addition to those sequence elements in the c-fos promoter homologous to previously defined cAMP-responsive elements, the SRE was also found to mediate cAMP responsiveness when attached to the c-fos core promoter $(-53$ to +42$)$. Whether this effect is mediated through the same SRE-binding factor (SRF; Prywes and Roeder 1987; Treisman 1987) that is believed to mediate induction by other agents (such as EGF, TPA, and insulin) or through a distinct factor remains to be established. It is possible that cAMP-mediated activation of transcription factors is a much more general phenomenon than previously thought, although clearly not all transcription factors are activated by cAMP. Further study of other cAMP-responsive genes should demonstrate whether this is, in fact, the case.

\section{Materials and methods}

\section{Cells and reagents}

$\mathrm{NIH}-3 \mathrm{~T} 3$ cells were grown in monolayer cultures in Dulbecco modified Eagle medium supplemented with $10 \%$ bovine calf serum. Stock was made of 8-bromo or dibutyrl cyclic AMP (Sigma), dissolved in distilled water at a concentration of $0.5 \mathrm{M}$. This $500 \times$ stock was added directly to the monolayer cultures to a final concentration of $1 \mathrm{~mm}$. A stock of 3-isobutyl l-methyl xanthine (IMX; Sigma) was dissolved in DMSO at a concentration of $0.5 \mathrm{M}(1000 \times$ stock $)$. This stock was then dissolved in serum-free medium as an $11 \times$ stock immediately before addition to the cells. One milliliter of the $11 \times$ stock was added per $10 \mathrm{ml}$ petri dish to a final concentration of $0.5 \mathrm{mM}$ IMX. Forskolin (Aldrich) was dissolved in $95 \%$ ethanol at a concentration of $10 \mathrm{~mm}$. This $1000 \times$ stock was added directly to monolayer cultures to a final concentration of $10 \mu \mathrm{M}$.

\section{Plasmid DNAs}

Many of the plasmids used in this study are diagramed in Figure 2. The construction of plasmids pFC700, pFC363, pFC72, pFC $\Delta 94 / 73$, pFC $\Delta 94 / 61, \mathrm{pTF} 1$, and pTF2 was described previously (Fisch et al. 1987). pFC72 was derived from pFC $\Delta 94 / 73$, an internal deletion mutant of pFC700 missing sequence be- tween -94 and -72 . pFC $\Delta 94 / 72$ was digested with XhoI $(-710)$ and $B g I I I(-72)$ and these sites were filled in with Klenow fragment of DNA polymerase I. The resulting large DNA fragment lacking sequence between -710 and -72 but containing the remaining sequence from $\mathrm{pFC700}$ was purified by gel electrophoresis and recircularized with T4 DNA ligase through its blunt ends. Both the XhoI and BglII sites were regenerated in the ligation.

pFC53 was derived from pFC $\Delta 94 / 53$ (Fisch et al. 1987), an internal deletion mutant of pFC700 missing sequence between -94 and -53 , exactly as described for $\mathrm{pFC72}$. The large fragment that was used to create pFC53 was also used to create pFC53E and pTF5/53, except that it was not filled in with Klenow. To create pFC53E and pTF5/53, this fragment was ligated through its cohesive ends to oligonucleotides TF4 or TF5, respectively. Oligonucleotide TF4 includes c-fos sequence from -319 to -297 and XhoI and BglII cohesive ends; oligonucleotide TF5 includes c-fos sequence from -303 to -281 and $X h o I$ and $B g I I I$ cohesive ends. The strands for these oligonucleotides were synthesized on an Applied Biosystems DNA synthesizer.

p700-225/53 and p225-99/53 were derived from pFC700 and pFC53 or pFC255 and pFC53, respectively. pFC700 was digested with $A p a I$; this site was filled in with the Klenow fragment and ligated to synthetic BglII DNA linkers. Digestion with $B g I I I$ and SstI removed excess linkers and generated a fragment including c-fos sequence from -700 to -225 and most of the pUC19 plasmid, exclusing polylinker sequence between the HindIII and SstI sites. This fragment was purified by gel electrophoresis and electroelution. pFC225 was digested with BstXI; this site was filled in with the Klenow fragment and ligated to synthetic BgIII DNA linkers. Digestion with BgIII and SstI removed excess linkers and generated a fragment including c-fos sequence from -225 to -99 and most of the pUC19 plasmid, excluding polylinker sequence between the HindIII and SstI sites. pFC53 was digested with BgIII and SstI to generate a fragment that included the c-fos and CAT sequence from pFC53 and the pUC19 polylinker between the HindIII and SstI sites. The latter fragment was ligated through its BgIII and SstI ends to either the fragment from $\mathrm{pFC700}$ or the fragment from pFC225.

p60LS was generated from pFC700, pFC $\Delta 94 / 61$ [an internal deletion mutant of pFC700 missing sequence between -94 and -61 (Fisch et al. 1987)], and a synthetic 36-base-pair oligonucleotide spanning positions -94 to -64 in c-fos with BssHII and $B g I I I$ cohesive ends. pFC700 was digested with BssHII and SstI to generate a fragment including c-fos sequence from -700 to -94 and the entire pUC19 plasmid, excluding polylinker sequence between the HindIII and SstI sites. pFC $\Delta 94 / 61$ was digested with BgIII and SstI to generate a fragment including c-fos sequence from -53 to +42 and the CAT gene. These two fragments were purified by gel electrophoresis and electroelution. The two fragments and the oligonucleotide were ligated together in a three-way ligation through their BssHII, BgIII, and SstI ends. Thus, p60LS is exactly analogous to pFC $\Delta 94 / 61$, except that in p60LS, c-fos sequence between -94 and -64 is regenerated by the oligonucleotide.

pFC $\Delta+10 /+42$ was derived from pFC363 and pFC700. pFC363 was digested with PstI; this site was blunted with the Klenow fragment and ligated to $X b a I$ DNA linkers. Digestion with $X b a I$ and $A p a I$ released excess linkers and generated a 235-base-pair fragment spanning positions -225 to +10 . pFC700 was digested with $A p a I$ and $X b a I$ to create a large fragment including c-fos sequence from -225 to -700 along with the CAT gene and the entire pUC19 plasmid. These fragments were purified by gel electrophoresis and electroelution and then ligated through their ApaI and XbaI cohesive ends. pFC $\Delta 94 / 30$, 
$\Delta-10 /+42$ was derived from pFC $\Delta 94 / 30$ (an internal deletion mutant of pFC700; Fisch et al. 1987) and pFC700. pFC $\Delta 94 / 30$ was digested with NarI; this site was blunted and ligated to $X b a I$ DNA linkers. Digestion with $X b a I$ and ApaI created a fragment including c-fos sequence from -10 to -30 and from -94 to -225 . This fragment was gel purified and ligated to the large ApaI to $\mathrm{XbaI}$ fragment from $\mathrm{pFC} 700$.

pTF4 was generated from pFC700 and the synthetic oligonucleotide TF4 described above. pFC700 was digested with ApaI; this site was filled in with the Klenow fragment and ligated to synthetic BgIII DNA linkers. Digestion with $B g I I I$ and XhoI released excess linkers and the $c$-fos promoter sequence between -700 and -225 . The synthetic oligonucleotide and the pFC700-derived fragment were ligated through their cohesive $X$ hoI and Bgll ends.

p21303-CAT (S.M. Hanly, P. Kim, A. Babich, and N. Heintz, in prep.) was a gift from Sarah Hanly. It contains sequence from -700 to +19 of the human histone H4A gene (Zhong et al. 1983) ligated to the CAT gene with its SV40 poly $(\mathrm{A})^{+}$site (and cloned into a pUC18 vector). A fragment spanning -48 to +19 in phuH4A and the first 500 base pairs of coding sequence from the CAT gene was prepared from p21303-CAT by digestion with AvaII (at -48 ) and NcoI. The Avall site was changed to a $B g I I I$ site by blunting with the Klenow fragment and ligating on BgIII synthetic DNA linkers. This fragment was used to generate pH4TATA and $\mathrm{pFC} 700-63 / \mathrm{H} 4$. To create pH4TATA, pFC53 was digested with $N c o I$ and BgIII. The large fragment including the pUC19 vector and CAT sequence downstream of the $\mathrm{NcOI}$ site was gel purified and ligated to the fragment from p21303-CAT. To create pFC700-63/H4, pFC60LS was digested with BglII and NcoI. The large fragment including c-fos sequence from -700 to -63 , CAT sequence downstream of the $N c o I$ site and the pUC19 vector was gel purified and ligated to the fragment from $\mathrm{p} 21303-\mathrm{CAT}$.

To generate pH4FC53, we gel purified an XmnI to AvaII fragment from $\mathrm{p} 21303-\mathrm{CAT}$. This fragment spanned -700 to -48 in the huH4A promoter and also included pUC18 sequence between $X m n I$ and HindIII sites. Prior to gel purification, the AvaII site had been blunted and ligated to BgIII DNA linkers. This fragment was ligated to an XmnI to BglII fragment from pFC53R, which is essentially the same as pFC53 but has the fosCAT sequence inserted in a pUC18 instead of a pUC19 vector. The $X m n I$ to $B g / I I$ fragment from pFC53R included the fosCAT sequence and pUC18 sequence between the $\mathrm{XmnI}$ and BamHI sites. pSVC63 was created by gel purifying a PVuII to NcoI fragment from pSV2neo (Southern and Berg 1982). This fragment was blunted with Klenow and BgIII linkers were added to both ends. The fragment was inserted into the BgIII site of pFC53R.

pTF6/H4 was generated from pFC700-63/H4 and an oligonucleotide (TF6) spanning positions -71 to -49 in $\mathrm{c}$-fos with $X h o I$ and $B g I I I$ cohesive ends. pFC700-63/H4 was digested with $X h o I$ and BglII to remove c-fos sequence between -700 and -63 . The large fragment was gel purified and ligated to the TF6 oligonucleotide.

pFC700SV, a mutant pFC700 containing the SV40 TATA box, was prepared by oligonucleotide-directed DNA repair of gapped heteroduplexes (Murinaga et al. 1984). Twenty micrograms of pFC700 plasmid DNA was split into two parts; one part was digested with $X m n I$ and treated with calf intestinal alkaline phosphatase (Boehringer Mannheim). The other part was digested with BssHII $(-99)$ and $\mathrm{XbaI}(+42)$. Digestion with $B s s \mathrm{HII}$ and $\mathrm{XbaI}$ generated a gap that served as the target region for mutagenesis. Three hundred nanograms each of these two plasmid preparations were mixed with a 500 -fold molar excess of the oligonucleotide 5'-CGCTTGTTATTTATGCAGTGGCT-3' and incubated at $100^{\circ} \mathrm{C}$ for $3 \mathrm{~min}, 30^{\circ} \mathrm{C}$ for $30 \mathrm{~min}, 4^{\circ} \mathrm{C}$ for 30 $\min$, and $0^{\circ} \mathrm{C}$ for $10 \mathrm{~min}$. Heteroduplex formation was confirmed by gel electrophoresis. Single-stranded gaps were repaired with Klenow. Escherichia coli (strain MC1061) transformants carrying the mutant plasmid were identified by colony hybridization (Grunstein and Hogness 1985) using the mutagenic oligonucleotide as a labeled probe. Mutant plasmid DNA was purified by retransformation and confirmed by dideoxy DNA sequencing (Sanger et al. 1977; Chen and Seeburg 1985).

pRSVCAT (Gorman et al. 1982) contains the long terminal repeat of Rous sarcoma virus fused to the CAT gene.

\section{Transfections}

$\mathrm{NIH}-3 \mathrm{~T} 3$ cells were plated $24 \mathrm{hr}$ prior to transfection at $1-1.5 \times 10^{6}$ cells per $10-\mathrm{cm}$ plate. The cells were refed with fresh medium $4 \mathrm{hr}$ before transfection by the calcium phosphate coprecipitation method (Wigler et al. 1977). Typically, 10 $\mu \mathrm{g}$ fosCAT plasmid, $5 \mu \mathrm{g}$ pRSVCAT plasmid (Gorman et al. 1982), and $10 \mu \mathrm{g}$ pUC19 carrier DNA were used per $10-\mathrm{cm}$ plate. The cells were exposed to transfection precipitate for 4 $\mathrm{hr}$, shocked with $15 \%$ glycerol in serum-free medium for $2 \mathrm{~min}$, washed twice with phosphate-buffered saline, and refed with medium containing $10 \%$ bovine calf serum. Twenty-four to twenty-eight hr after transfection, the cells were treated with 1 $\mathrm{mM}$ 8-Br cAMP for $30 \mathrm{~min}$. The cells were then washed twice with ice-cold phosphate-buffered saline and lysed with $2.5 \mathrm{ml}$ of urea lysis buffer [ $7 \mathrm{M}$ urea, $2 \%$ sarcosyl, $350 \mathrm{~mm} \mathrm{NaCl}, 10$ mM Tris $[\mathrm{pH} 7.9 \mid, 1 \mathrm{~mm}$ EDTA]. The lysed cells were homogenized by 20 strokes of a glass Dounce homogenizer /type B pestle), and total cellular RNA was isolated by centrifugation of the homogenized lysates in CsCl gradients (Glisin et al. 1974). RNA pellets were dissolved in water and stored as ethanol precipitates. When serum starvation was carried out, the transfected cells were incubated for 24 to $28 \mathrm{hr}$ in medium containing $0.5 \%$ bovine calf serum starting at $\sim 16 \mathrm{hr}$ after transfection. In this case, total cellular RNA was harvested 40 to 44 hr after transfection.

\section{RNA analysis}

Mapping a transfected fosCAT fusion gene transcripts by S1 nuclease analysis was performed as described previously (Fisch et al. 1987). The S1 probe was an $\sim 400$-bp fragment derived from pFC700 that spanned positions -99 to +42 in c-fos and the first $250 \mathrm{bp}$ of coding sequence from the CAT gene, along with a small intervening segment of pUC polylinker. pFC700 was digested with EcoRI enzyme (Boehringer Mannheim Biochemicals), dephosphorylated with calf intestinal alkaline phosphatase, and labeled with T4 polynucleotide kinase (New England Biolabs $)$ and $\left[\gamma^{32} \mathrm{P}\right] \mathrm{ATP}$. Secondary digestion with $B s s H I I$ enzyme (New England Biolabs) yielded a labeled 400-bp fragment that was purified by electrophoresis through a $1 \%$ agarose gel followed by electroelution and concentration in a Schleicher and Schuell Elu-trap.

The $S 1$ probe used for mapping transcripts from $\mathrm{pFC} \Delta+10$ / +42 was derived from that plasmid by labeling the EcoRI site in CAT with $\left[\gamma^{32}\right.$ P $]$ ATP and then secondarily digesting with ApaI to generate an $\sim 490$-bp labeled fragment. This fragment thus included 250 base pairs of CAT coding sequence and c-fos sequence from -225 to +42 . The labeled fragment was purified as described above.

The $\mathrm{S} 1$ probe used for mapping transcripts derived from chimeric c-fos/huH4A-CAT transcripts was derived from pFC700$63 / \mathrm{H} 4$. This plasmid was labeled with $\left[\gamma^{-32} \mathrm{P}\right] \mathrm{ATP}$ at the $E c o$ RI site in CAT and then secondarily digested with BssHII enzyme 
to generate a labeled fragment $\sim 360 \mathrm{bp}$ in length. Thus, this fragment included 250 base pairs of CAT coding sequence, huH4A sequence from -48 to +19 and $c$-fos sequence from -100 to -63 . The labeled fragment was purified as described above. Hybridization of the probes to total cellular RNA was performed overnight $(>12 \mathrm{hr})$ at $44^{\circ} \mathrm{C}$. S1 digestion was performed for $30 \mathrm{~min}$ at $37^{\circ} \mathrm{C}$ with $37.5 \mathrm{U}$ of S1 nuclease (Boehringer Mannheim Biochemicals) per 200- $\mu$ l reaction. Nuclease-resistant fragments were fractionated on $5 \%$ polyacrylamide-urea $7 \mathrm{M}$ gels.

Transcript mapping by RNase protection was performed as described by Zinn et al. (1983). For mapping endogenous murine c-fos mRNA, a 385-bp complementary strand RNA probe was generated from an SP65 plasmid containing a $1 \mathrm{~kb} E c 0 \mathrm{RI}$ to HindIII fragment from the mouse c-fos gene that spanned the first exon. This plasmid was kindly provided by Tom Curran. In the RNase protection assay this probe generated a 286-bp fragment characteristic of properly initiated c-fos mRNA.

The levels of induction as determined by S1 mapping and RNase protection were quantitated with a Beckman DU8-B scanning densitometer. Multiple exposures of autoradiograms were obtained to assure that all lanes of any given gel would be represented in an exposure within the linear response range of the film (Kodak XAR-5 or BB-5) and of the densitometer. In the figures shown, one or two of these exposures were chosen for presentation.

\section{Acknowledgments}

We would like to thank Virginia F. Kozler for excellent technical assistance with the cell culture. This work was supported by Public Health Service grant CA42567 from the National Institutes of Health to R.G.R. and by general support from the Pew Trusts. T.F. is supported in part by MSTP grant GM00739. R.P. is a Fellow of the Jane Coffin Child Memorial Fund for Medical Research.

\section{References}

Angel, P., M. Imagawa, R. Chiu, B. Stein, R.J. Imbra, H.J. Rahmsdorf, C. Jonat, P. Herrlich, and M. Karin. 1987. Phorbol ester-inducible genes contain a common cis element recognized by a TPA-modulated trans-acting factor. Cell 49: 729-739.

Bravo, R., M. Neuberg, J. Burckhardt, J. Almendral, R. Wallich, and R. Muller. 1987. Involvement of common and cell typespecific pathways in c-fos gene control: Stable induction by cAMP in macrophages. Cell 48: 251-260.

Chen, E.Y. and P. Seeburg. 1985. Supercoil sequencing: A fast and simple method for sequencing plasmid DNA. DNA 4: $165-170$.

Chiu, R., M. Imagawa, R. Imbra, J.R. Bockoven, and M. Karin. 1987. Multiple cis- and trans-acting elements mediate the transcriptional response to phorbol esters. Nature 329: 648651.

Comb, M., N.C. Birnberg, A. Seasholtz, E. Herbert, and H.M. Goodman. 1986. A cyclic AMP- and phorbol ester-inducible DNA element. Nature 323: 353-356.

Curran, T. 1988. fos. In The oncogene handbook (ed. E.P. Reddy, A.M. Skalka, and T. Curran). pp. 305-325. Elsevier, Amsterdam.

Dailey, L., S.M. Hanly, R.G. Roeder, and N. Heintz. 1986. Distinct transcription factors bind specifically to two regions of the human histone H4 promoter. Proc. Natl. Acad. Sci. 83: $7241-7245$.

Distel, R.J., B.S. Rosen, and B.M. Spiegelman. 1987. Nucleoprotein complexes that regulate gene expression in adipocyte differentiation: Direct participation of c-fos. Cell 49: 835844.

Fisch, T.M., R. Prywes, and R.G. Roeder. 1987. c-fos sequences necessary for basal expression and induction by epidermal growth factor, 12-O-tetradecanoyl phorbol-13-acetate, and the calcium ionophore. Mol. Cell. Biol. 7: 3490-3502.

Franza, B.R., Jr., F.R. Rauscher III, S.F. Josephs, and T. Curran. 1988. The fos complex and fos-related antigens recognize sequence elements that contain the AP-1 binding sites. Science 239: 1150-1153.

Gilman, M.Z. 1988. The c-fos serum response element responds to protein kinase $\mathrm{C}$-dependent and -independent signals, but not to cyclic AMP. Genes Dev. 2: 394-402.

Gilman, M.Z., R.N. Wilson, and R.A. Weinberg. 1986. Multiple protein-binding sites in the 5 ' flanking region regulate c-fos expression. Mol. Cell. Biol. 6: 4305-4316.

Glisin, V., R. Crkvenjakov, and C. Byus. 1974. Ribonucleic acid isolation by cesium chloride centrifugation. Biochemistry 13: $2633-2637$.

Gorman, C.M., G.T. Merlino, M.C. Willingham, I. Pastan, and B.H. Howard. 1982. The Rous sarcoma virus long terminal repeat is a strong promoter when introduced into a variety of eukaryotic cells by DNA-mediated transfection. Proc. Natl. Acad. Sci. 79: 6777-6781.

Greenberg, M.E., L.A Greene, and E.B. Ziff. 1985. Nerve growth factor and epidermal growth factor induce rapid transient changes in proto-oncogene transcription in $\mathrm{PCl} 2$ cells. $/$. Biol. Chem. 260: 14101-14110.

Greenberg, M.E., Z. Siegfried, and E.B. Ziff. 1987. Mutation of the c-fos gene dyad symmetry element inhibits serum inducibility of transcription in vivo and the nuclear regulatory factor binding in vitro. Mol. Cell. Biol. 7: 1217-1225.

Grunstein, M. and D.S. Hogness. 1985. Colony hybridization: A method for the isolation of cloned DNAs that contain a specific gene. Proc. Natl. Acad. Sci. 72: 3961-3965.

Hardy, S. and T. Shenk. 1988. Adenovirus control regions activated by E1A and the cAMP response element bind to the same factor. Proc. Natl. Acad. Sci. 85: 4171-4175.

Hurst, H.C. and N.C. Jones. 1988. Identification of factors that interact with the E1A-inducible adenovirus E3 promoter. Genes Dev. 1: 1132-1146.

Imagawa, M., R. Chiu, and M. Karin. 1987. Transcription factor AP-2 mediates induction by two different signal-transduction pathways: Protein kinase C and cAMP. Cell 51: 251260.

Krebs, E.G. and J.R. Beavo. 1979. Phosphorylation-dephosphorylation of enzymes. Annu. Rev. Biochem. 48: 923-959.

Kruijer, W., D. Schubert, and I.M. Verma. 1985. Induction of the proto-oncogene fos by nerve growth factor. Proc. Natl. Acad. Sci. 82: 7330-7334.

Lee, K.A.W. and M.R. Green. 1987. A cellular transcription factor E4F1 interacts with an Ela-inducible enhancer and mediates constitutive enhancer function in vitro. EMBO $\mathrm{J}$. 6: $1345-1353$.

Lee, W., P. Mitchell, and R. Tjian. 1987. Purified transcription factor AP-1 interacts with TPA-inducible enhancer elements. Cell 49: 741-752.

Lin, Y.S. and M.R. Green. 1988. Interaction of a common cellular transcription factor, ATF, with regulatory elements in both Ela- and cAMP-inducible promoters. Proc. Natl. Acad. Sci. 85: 3396-3400.

Mermod, N., T.J. Williams, and R. Tjian. 1988. Enhancer binding factors AP-4 and AP-1 act in concert to activate SV40 late transcription in vitro. Nature 332: 557-561.

Montminy, M.R., K.A. Sevarino, J.A. Wagner, G. Mandel, and R.H. Goodman. 1986. Identification of a cyclic-AMP-re- 
sponsive element within the rat somatostatin gene. Proc. Natl. Acad. Sci. 83: 6682-6686.

Montminy, M.R. and L.M. Bilezikjian. 1987. Binding of a nuclear protein to the cyclic-AMP response element of the somatostatin gene. Nature 328: 175-178.

Murinaga, Y., T. Franceschini, S. Inouye, and M. Inouye. 1984. Improvement of oligonucleotide-directed site-specific mutagenesis using double-stranded plasmid DNA. Biotechnology 2: 636-639.

Nishizuka, Y. 1986. Studies and perspectives of protein kinase C. Science 233: 305-312.

Piette, J. and M. Yaniv. 1987. Two different factors bind to the $\alpha$-domain of the polyoma virus enhancer, one of which also interacts with the SV40 and the c-fos enhancers. EMBO $J$. 6: $1331-1337$.

Prywes, R. and R.G. Roeder. 1987. Purification of the c-fos enhancer binding protein. Mol. Cell. Biol.7: 3482-3489.

Rauscher, F.J. III, L. Sambucetti, T. Curran, R.J. Distel, and B.M. Spiegelman. 1988a. Common DNA binding site for fos protein complexes and transcription factor AP-1. Cell 52: 471480.

Rauscher, F.J. III, D.R. Cohen, T. Curran, T.J. Bos, P.K. Vogt, D. Bohmann, R. Tjian, and B.R. Franza, Jr. 1988b. fos-associated protein 39 is the product of the iun proto-oncogene. Science 240: 1010-1016.

Sanger, F., S. Nicklen, and A.R. Coulson. 1977. DNA sequencing with chain terminating inhibitors. Proc. Natl. Acad. Sci. 74: 5463-5467.

Setoyama, C., G. Frunzio, G. Lian, M. Mudryi, and B. de Crombrugghe. 1986. Transcriptional activation encoded by the v-fos gene. Proc. Natl. Acad. Sci. 83: 3213-3217.

Simon, M.C., T.M. Fisch, B.J. Benecke, J.R. Nevins, and N. Heintz. 1988. Definition of multiple, functionally distinct TATA elements, one of which is a target in the hsp70 promoter for E1A regulation. Cell 53: 723-729.

SivaRaman, L., S. Subramanian, and B. Thimmappaya. 1986. Identification of a factor in HeLa cells specific for an upstream transcriptional control sequence of an Ela-inducible adenovirus promoter and its relative abundance in infected and uninfected cells. Proc. Natl. Acad. Sci. 83: 5914-5918.

Southern, P.J. and P. Berg. 1982. Transformation of mammalian cells to antibiotic resistance with a bacterial gene under control of the SV40 early region promoter. I. Mol. Appl. Genet. 1: 327-341.

Stumpo, D.J., T.N. Steward, M.Z. Gilman, and P.J. Blackshear. 1988. Identification of $\mathrm{c}$-fos sequences involved in induction by insulin and phorbol esters. J. Biol. Chem. 263: 16111614.

Treisman, R. 1985. Transient accumulation of c-fos RNA following serum stimulation requires a conserved 5 ' element and c-fos 3' sequences. Cell 42: 889-902.

. 1987. Identification and purification of a polypeptide that binds to the c-fos serum response element. EMBO $/$. 6: 2711-2717.

Tsukada, T., J.S. Fink, G. Mandel, and R.M. Goodman. 1987. Identification of a region in the human vasoactive intestinal polypeptide gene responsible for regulation by cyclic AMP. J. Biol. Chem. 262: 8743-8747.

Wigler, M., S. Silverstein, L.S. Lee, A. Pellicer, Y.-C. Cheng, and R. Axel. 1977. Transfer of purified herpes virus thymidine kinase gene to cultured mouse cells. Cell 11: 223-232.

Zhong, R., R.G. Roeder, and N. Heintz. 1983. The primary structure and expression of four cloned histone genes. $\mathrm{Nu}$ cleic Acids Res. 11: 7409-7425.

Zinn, K., D. DiMaio, and T. Maniatis. 1983. Identification of two distinct regulatory regions adjacent to the human $\beta$-interferon gene. Cell 34: 865-879. 


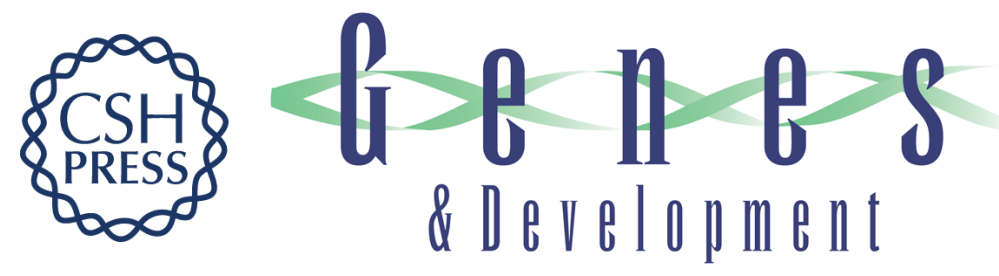

\section{Multiple sequence elements in the c-fos promoter mediate induction by CAMP.}

T M Fisch, R Prywes, M C Simon, et al.

Genes Dev. 1989, 3:

Access the most recent version at doi:10.1101/gad.3.2.198

References This article cites 45 articles, 20 of which can be accessed free at:

http://genesdev.cshlp.org/content/3/2/198.full.html\#ref-list-1

\section{License}

Email Alerting

Service

Receive free email alerts when new articles cite this article - sign up in the box at the top right corner of the article or click here.

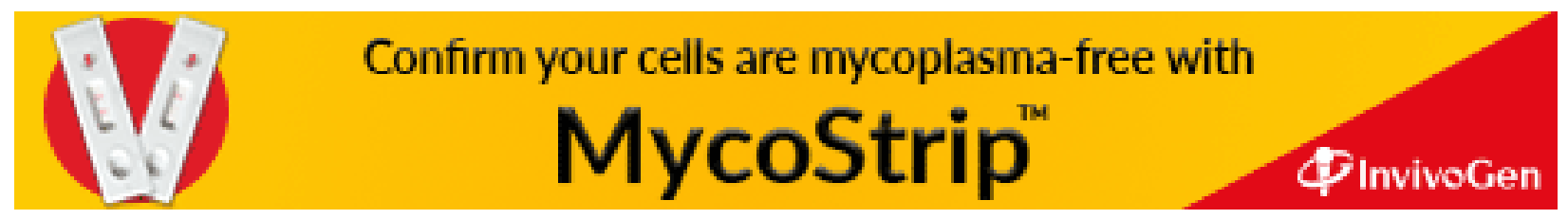

